\section{RELATIVITY}

\section{Signature of a Black Hole}

by our Cosmology Correspondent

IN spite of all the fuss about X-ray stars in binary systems and the difficulty of explaining the presence of these sources by any simple astrophysical model, the case that these systems contain black holes is still not proven. Certainly it is true that in some cases, at least, there is good evidence that the underlying object associated with the X-ray emission is both dark, and thus presumably too cool for nuclear burning to be holding it up against gravitational pressure, and also that the object is more massive than the limit above which a cool, nonrotating object will collapse into a singularity without such support. But recently two groups have independently pointed out that the compact X-ray sources might be associated with differentially rotating degenerate dwarfs (Brecher and Morrison, Astrophys. J. Lett., 180, L107 ; 1973 ; and Lamb and Van Horn, Astrophys. J., 183, 959 ; 1973).

Lamb and Van Horn, for example, have constructed a detailed, semi-quantitative model of such a star which has a mass of 1.2 to $1.5 \mathrm{M}_{\odot}$ and can explain all the observed properties of Her X-1. It is known that degenerate dwarfs exist, and there seems every reason to believe that they will rotate, perhaps differentially. So where is the need to introduce such mathematical curiosities as black holes? Until all simpler explanations of these compact X-ray sources are eliminated, the relativists must look elsewhere for the conclusive proof that these singular predictions of their theories are correct.

Two astrophysicists who have done just that are Cunningham and Bardeen. They have calculated the detailed effect of a Kerr metric black hole on radiation emitted from a star of $1 M_{\odot}$ which is in a circular orbit about the hole. A combination of Doppler shifts, gravitational redshifts and gravitational focusing combines to modulate the light from the star, as seen by a distant observer; the first two effects determine the surface brightness of the image seen, the third determirles its size (Astrophys. J., 183, 237 ; 1973).

The problem is far from trivial. But its solution is worth the effort involved because it suggests a means for detecting black holes which is specific to them. No other system could produce a similar light curve, and as an added incentive if such a system were discovered by this signature the detailed variations of brightness and of the image size would provide a test of the strong-field predictions of general relativity.

A related investigation of this effect has been carried out by Maeder
(Astron. Astrophys., 26, 215; 1973). Both calculations show that the influence of black holes on companion stars should be detectable by present telescopes, and they should lead to an effort by observers to find such peculiarly varying systems.

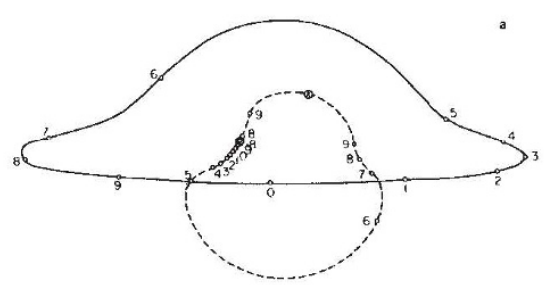

Apparent position of the two brightest images $(-; .--)$ as functions of time for one particular orbital radius and an observer at a polar angle of $84.24^{\circ}$.

Numbered intervals define ten equally spaced times.

What should the observers look for? The actual light curve seen, of course, depends on the orientation of the orbital plane of the black hole/star system relative to the observer. In addition, the images produced will move around the sky as the star orbits the black hole and its light is bent in different directions. And as a third mark of the presence of a black hole in such a system, more than one image is produced (see figure). It might not be possible to resolve the multiple images, which is a pity; but if there is a black hole at the centre of our Galaxy, as some people suggest, then there might well be a good chance of observing this kind of effect. Cunningham and Bardeen suggest in particular that the radio signal from a pulsar orbiting a black hole of some $10^{6} M_{\circlearrowleft}$ at the galactic centre would show the required modulation. Extinction along the line of sight to the nucleus makes optical observation impossible, but the pulsar period would be so small compared with the orbital period of the neutron star about the black hole that the radio technique would be quite practicable.

Maeder prefers to concentrate on effects which might be produced when a black hole of a few solar masses and a star form a binary system, and he predicts that in some circumstances observers on Earth might see a brightening amounting to several tenths of a magnitude and lasting for several hours. In either case, these models and the observable effects they predict provide a much more realistic attack on the problem of the reality or otherwise of black holes than does the approach of invoking such singularities to account for observations which can also be explained by less extreme models.

\title{
Acylated tRNA Metamorphoses
}

Sprinzel and Cramer, writing in Nature New Biology next Wednesday (September 5), suggest that tRNA is aminoacylated exclusively in the 2 -hydroxyl position, but that the aminoacyl group is transferred to the $3^{\prime}-\mathrm{OH}$ position before the reaction of peptidyl tRNA with aminoacyl tRNA in the ribosome.

Evidence for the isomerization of acylated tRNA during protein biosynthesis comes from the study of analogues of tRNA ${ }^{\text {Phe }}$ lacking either the 2'- or $3^{\prime}$-OH group in the $3^{\prime}$-terminal adenosine. Only the tRNA ${ }^{\text {Phe }}-\mathrm{C}-\mathrm{C}-3^{\prime} \mathrm{dA}$ can accept phenylalanine, exhibiting the same $K_{m}$ and only a slightly lower $V_{\max }$ compared with normal tRNA ${ }^{\mathrm{Ph}}-\mathrm{C}-\mathrm{C}-\mathrm{A}$. tRNA $^{\text {Phe }}{ }_{-} \mathrm{C}-\mathrm{C}-2^{\prime} \mathrm{dA}$ is, however, a competitive inhibitor of the aminoacylation reaction. Such structurally-related molecules as periodate-oxidized tRNA $^{\text {Pho }}$ tRNA ${ }^{\text {Pho }}-\mathrm{C}-\mathrm{C}-\mathrm{A}_{\mathrm{oxi}}$ and tRNA $^{\text {Phe }}-\mathrm{C}-\mathrm{C}$ are not competitive inhibitors.

Sprinzel and Cramer conclude that, although tRNA ${ }^{\text {Phe }}$-C-C-2'dA can be bound to the active site, aminoacylation can occur only when the hydroxyl group is in the $3^{\prime}$ position.

In normal tRNA, acyl migration between $2^{\prime}$ and $3^{\prime} \mathrm{OH}$ occurs with a half time of $0.3 \mathrm{~s}$. Thus peptidyl tRNA might donate its acyl group to the incoming aminoacyl tRNA by way of only one specific or either by way of both of these two positions on the ribose ring. Sprinzel and Cramer's evidence suggests that the ribose $2^{\prime}-\mathrm{OH}$ group is the sole effective acyl donor.

First, they cite earlier evidence that the $2^{\prime}$ analogue of puromycin is inactive as an inhibitor of protein synthesis. This suggests that the $3^{\prime}$-peptidyl tRNA isomer is the only acyl donor in vivo. Their own experimental evidence is consistent with this view because they find that Phe-tRNA-C-C-3'dA cannot be utilized in a poly(U)-directed peptide synthesizing system in vitro. Free PhetRNA ${ }^{\text {Phe }}-\mathrm{C}-\mathrm{C}-3^{\prime} \mathrm{dA}$ on the other hand can undergo hydrolysis (although three times slower than the normal tRNA). Transacylation must therefore be an obligatory step in protein synthesis.

This work suggests that there may be highly stringent steric requirements for the ribose isomers for both aminoacylation of tRNA by the twenty or so different synthetase enzymes and for formation of the peptide bond catalysed by peptidyl synthetase in the active site of the ribosome. 